\title{
Physical activity and sedentary lifestyle in children as time-limited functions: Usefulness of the principal component analysis method
}

\author{
Comlavi B. Guinhouya, Stephane Soubrier, Christian Vilhelm, \\ Pierre Ravaux, Mohamed Lemdani, Alain Durocher, and Hervé Hubert \\ University of Lille 2, Loos, France
}

\begin{abstract}
This study was designed to examine the hourly variation in and the interplay between physical activity and sedentary behavior (SB) in order to highlight key time periods for physical activity interventions for children. Data for physical activity and SB obtained with ActiGraph in 56 boys and 47 girls aged from 8 to 11 years. These data were divided into sixty minute-time samples for moderate-to-vigorous physical activity (MVPA) and $\mathrm{SB}$, and analyzed using a principal component analysis (PCA) and correlation statistics. The PCA provides 10 factors which account for $80.4 \%$ of the inertia. Only two of these factors did not display competition between MVPA and SB. Contrary to some reports, a coefficient of correlation of $-.68\left(p<10^{-4}\right)$ was found between daily time spent at MVPA and SB. Some salient traits of children's behaviors were shown through PCA. The results suggested that efficacy of interventions targeting the morning hours (07:00 AM-11:59 AM) and the afternoon period (02:00 PM-05:59 PM) warrants attention.
\end{abstract}

There is a growing recognition that behavioral and environmental factors are determining the alarming increase of obesity in children (Rennie, Johnson, \& Jebb, 2005). The pair physical activity/physical inactivity is seen to be the most relevant way for developing prevention or treatment strategies toward pediatric population; in that restricting food intake in the growing body of a child must be discouraged. It is believed that moderate-to-vigorous physical activity (MVPA) is required to produce health benefits, whereas sedentary behaviors (SB) or sustained physical inactivity may be risk factors for obesity in youth (Dietz, 1996). Despite the substantial body of research by Epstein and colleagues (Epstein, Paluch, Gordy, \& Dorn, 2000; Epstein, Saelens, Myers, \& Vito, 1997; Epstein, Saelens, \& O’Brien, 1995; Epstein, Smith, Vara, \& Rodefer, 1991) showing that a reduction in physical inactivity among children may translate into an increase of physical activity and reduce obesity, these two sets of behavior are viewed as independent and analyzed as such. For example, Fox (2004) stated that physical activity and sedentary lifestyle cannot be regarded as two sides of the same coin. To account for this distinction, some authors mentioned the low relationship between the two behaviors (Marshall, Biddle, Sallis, McKenzie, \& Conway, 2002). Nevertheless, only some types of activity categories deemed to represent children's sedentary habits were used for comparison. For instance, Biddle, Gorely, and Stensel (2004) and Biddle and Gorely (2005) found that researchers usually assess the "absence of activity" rather than the
SB of children, arguing that previous definitions of physical inactivity or sedentary behavior did not tell anything about what inactive people are actually doing. However, if doing so for SB, it should be the same approach for physical activity in order to be able to cross match dimensions (e.g., a type of SB compared to a type of physical activity: television viewing vs. soccer playing with pairs) of these two sets of behaviors. In addition, few studies highlighted the discrepancies in methods used to analyze each of them. Physical activity is increasingly assessed with objective tools, such as accelerometry, when SB is often subjectively expressed as the time spent in front of television and related attitudes (video, video game playing, computer). In a cross-national study, Marshall et al. (2002) reported however that SB is complex and cannot be accurately captured by only television watching.

Due to technological progress, SB has become objectively quantifiable in children using accelerometry (Reilly et al., 2003). This technique does not specify the type or the context of the SB but can evaluate the level of its associated movement. It can be hypothesized that SB and physical activity are competing behaviors which vary as a function of the limited number of hours in the day (Jago, Baranowski, Thompson, Baranowski, \& Greaves, 2005). Nonetheless, to the best of our knowledge, no study has addressed the interplay between activity and sedentary lifestyle as a whole. The question of specific time periods where inactivity counterbalances activity is not yet well documented. Such an approach can help health pro-

H. Hubert, herve.hubert@univ-lille2.fr 
moters to refine strategies leading to physical activity enhancement.

It is the aim of this study to examine the hourly variation in and the interplay between physical activity and SB among children. Principal component analysis (PCA) is a multidimensional statistical method which uses parallel analysis technique to highlight the significance of specific units within a whole. Applying this analysis technique to hourly cluster data of physical activity and SB might help to get insight on some key periods of interaction which can be used in prevention strategies. The adequacy of such an approach will then be discussed.

\section{METHOD}

\section{Sample and Setting}

One hundred and three pupils ( 56 boys and 47 girls) were randomly selected from three elementary schools of the Lille school district (France). Children aged from 8 to 11 years were invited to participate in this study. The mean age was $9.93 \pm 0.80$ years. The mean height and weight were $1.37 \pm 0.07 \mathrm{~m}$ (male, $1.39 \pm 0.06 \mathrm{~m}$; female, $1.39 \pm 0.08 \mathrm{~m}$ ) and $35.6 \pm 7.4 \mathrm{~kg}$ (male, $35.7 \pm$ $7.0 \mathrm{~kg}$; female, $35.6 \pm 8.0 \mathrm{~kg}$ ), respectively. This exploratory design received the approval of the local advisory board (ethical committee for the protection of persons in biomedical research of Lille). Prior to the beginning of the study, children and their parents provided written informed consent. Children were apparently healthy with no declared physical activity-related disease. However, $33 \%$ of the children (32\% of male and $34 \%$ of female) were considered as overweight/obesity according to the sex- and age-specific BMI cutoff points proposed by the International Obesity Task Force (Cole, Bellizi, Flegal, \& Dietz, 2000). Children's height was measured with a wall stadiometer (Vivioz Medical, Paris) and their body mass was assessed by a calibrated balance (Tanita BC532, Tanita Inc., Tokyo).

\section{Procedure and Instrumentation}

Behaviors were quantified with uniaxial accelerometers (MTI-ActiGraph, Model 7164, Florida, USA) during 3 consecutive days ( 2 school days: Monday and Tuesday + 1 free day: Wednesday). It has previously been shown that a measurement period of 2 school days (including Monday) and one free day may be a reasonable compromise for the study of habitual physical activity (HPA) in children (Falgairette, Gavarry, Bernard, \& Hebbelinck, 1996). Accelerometer was tightly mounted at the right hip of each child at the beginning of the measurements by an evaluator. Every morning, the same evaluator verified if there were any problem with the activity monitor and reported information about any physical activity performed (or any moment) while the monitor could not be worn (e.g., swimming, bath).

This activity monitor measures $5.1 \times 3.8 \times 1.5 \mathrm{~cm}$, is lightweight $(42 \mathrm{~g})$, and is powered by a readily available 2430 coin cell lithium battery. The device is a uniaxial monitor which integrated accelerations/decelerations in the vertical plane via a piezoelectric plate. Acceleration detection ranges in magnitude from 0.05 to $2.00 \mathrm{G}$, and the frequency responses range from 0.25 to $2.5 \mathrm{~Hz}$, so that motion outside normal human movement is rejected by a filtered bandpass. The acceleration-deceleration signal is digitized by an analog-to-digital converter and numerically integrated over a user-defined time interval (epoch). The rate of change of acceleration is sampled 10 times per second and the data are summed up into time per epoch and stored in the internal memory; then the numerical integrator is reset. To begin data collection, monitors are initialized using a compatible personal computer (PC). A real-time internal clock allows the researcher to begin collecting at desired time. The outputs from ActiGraph monitor are expressed in "counts" per each epoch, which are equivalent to the amount and magnitude of acceleration summed up during each epoch. That is, higher numbers represent a combination of higher frequency and intensity of movement during a given epoch. For the purpose of the current study, accelerometers were initialized to capture movement counts within 1-min intervals which allow the device to collect 22 consecutive days of data (Actisoft analysis software, user's manual, www .theactigraph.com).

\section{Data Reduction}

Data collected over the 3 days were pooled together for analyses. Raw data were processed with an Excel macro. The time spent at MVPA $\left(t_{\text {MVPA }}\right)$ of each day was determined according to Puyau, Adolph, Vohra, and Butte (2002). The mean value across the three was retained as the overall $t_{\text {MVPA }}$ of each child. Puyau et al. (2002) calibrated and validated ActiGraph counts against 6-h energy expenditure in children aged from 6 to 16 years old. A cutoff for MVPA was then developed as activity counts of $\geq 3,200$ counts per minute (cpm). On the other hand, the time spent in $\mathrm{SB}\left(t_{\mathrm{SB}}\right)$ was defined according to the $1,100 \mathrm{cpm}$ of Reilly et al. (2003). In a study aiming specifically on objective measurement of SB, Reilly et al. (2003) developed the cutoff of $1,100 \mathrm{cpm}$ in young children aged from 3 to 4 years old. Its cross-validation provides a specificity of $82 \%$ and sensitivity of $83 \%$.

No data of less than 10 hours of monitoring per day were included in the study. Daily totals for MVPA and SB were calculated by summing the value from $14 \mathrm{~h}$ (07:00 AM and 09:00 PM) with 60-min time blocks comprising each day. The daily $t_{\mathrm{MVPA}}$ and $t_{\mathrm{SB}}$ were categorized in four periods: morning (07:00 AM-11:59 AM), noon (12:00 PM-01:59 PM), afternoon (02:00 PM-05:59 PM), and evening (06:00 PM-09:00 PM).

\section{Statistical Analysis}

Data are displayed as mean \pm standard deviation. Between-day intraclass reliability coefficients ( $R$ and $95 \%$ confidence intervals [CIs]) were computed using repeated measure ANOVA to determine the stability of the accelerometer counts across the three days of assessment. The single measurement reliability was also computed. Gender differences were examined with independent Student 
$t$ tests and a chi-square test. Correlation analyses were carried out by a Pearson Product moment coefficients of correlation. The level of significance was set at $p<.05$. The patterns of physical activity and sedentary behavior were described using $t_{\mathrm{MVPA}}$ and $t_{\mathrm{SB}}$ totals from each 60min block between 07:00 AM and 09:00 PM. These data sets were subjected to a principal component analysis (PCA) with varimax rotation. PCA is a statistical technique that converts a large number of related variables into a smaller set of "factors" or "components" intended to describe specific patterns of behavior. The weights (factor loadings) or coefficients are selected to render each factor independent of the others and to sequentially explain the largest amount of the total variance possible. Whereas this analytical model was introduced to nutritional epidemiology as an attempt to capture the multidimensionality of diet while reducing the apparent complexity (McCann, Weiner, Graham, \& Freudenheim, 2001; Randall, Marshall, Graham, \& Brasure, 1991; Ursin et al., 1993), it appears to be underused in the field of physical activity. Taking into account the large variability of activity behaviors (e.g., physical activity and sedentary behavior) in a week, a day, an hour, such an analytic technique may be adequate to subtract the underlying temporal structure of the variance. In the present study the averaged 60 -min block periods monitored during the three days served analysis. Only factors with an eigenvalue $\geq 1$ were retained.

\section{RESULTS}

As shown in Table 1, there were no significant difference between boys and girls for either anthropometric characteristics $(p>.05)$ or for their status with respect to overweight/obesity $\left(\chi^{2}=0.042, p=.838\right)$. However, girls spent more time in SB than boys $(p<.01)$. A reverse trend was obtained for $t_{\mathrm{MVPA}}(p<.0001)$.

\section{Reliability Coefficients}

As shown in Table 2, the single-day reliability of $t_{\text {MVPA }}$ and $t_{\mathrm{SB}}$ were $0.50(95 \% \mathrm{CI}: 0.39,0.61)$ and 0.35 (95\% CI: $0.23,0.48)$, respectively. Single-day reliability represents the reliability of the data obtained on 1 day, if only 1 day of measurement was available and serves as a point of comparison for other all days. These values were comparable to those reported in previous studies (Mota, Santos, Guerra, Ribeiro, \& Duarte, 2003; Trost, Pate, Freedson, Sallis, \& Taylor, 2000). The average of the 3 days' measurement coefficients of reliability were of 0.75 (95\% CI: $0.66,0.82)$ and $0.62(95 \% \mathrm{CI}: 0.48,0.73)$ for $t_{\mathrm{MVPA}}$ and $t_{\mathrm{SB}}$, respectively. This result shows that pooling data together make them suitable for analysis since reliability coefficients are enhanced. The 3-day data seem to be adequate with regard to a coefficient of reliability of 0.77 reported by Trost et al. (2000) for 4 days of monitoring.

\section{Time Period Analysis, Relationship and Variance in MVPA, and SB Along Days}

From the 28 items included, 10 components were extracted (Table 3), which accounted for $80.4 \%$ of the total inertia. Within each time component, children's participation in $t_{\mathrm{MVPA}}$ and $t_{\mathrm{SB}}$ was consistent, yet distinct from other time segment.

A coefficient of correlation of $-0.68\left(p<10^{-4}\right)$ was found between daily $t_{\mathrm{MVPA}}$ and $t_{\mathrm{SB}}$. When examining this relationship over hours, it appears that except from a morning hour (08:00 AM-08:59 AM) which showed no significant correlation between $t_{\mathrm{MVPA}}$ and $t_{\mathrm{SB}}$, there were high significant and negative coefficients of correlation between $t_{\mathrm{MVPA}}$ and $t_{\mathrm{SB}}(-.81 \leq r \leq-.64)$. Highest coefficients of correlation were observed at 09:00-09:59 and 04:00-04:59; and the lowest coefficients of correlation were obtained at 07:00-07:59 and 02:00-02:59.

The relative participation in MVPA and SB (percentage of time in MVPA or SB related to the total amount of daily $t_{\mathrm{MVPA}}$ and $t_{\mathrm{SB}}$ ) are presented in Figures 1 and 2. For MVPA, no clear patterns or differences among genders were obtained. Nonetheless, on average, girls and boys spent the highest percentage of their MVPA-time in the afternoon (02:00 PM-05:59 PM, 42.3\% and 45.2\% of the total daily MVPA for girls and boys, respectively). On the other hand, the highest percentage they spent being sedentary occurred in the morning hours (07:00 AM-11:59 AM, $37.6 \%$ and $38.3 \%$ for girls and boys, respectively). Furthermore, in the afternoon, boys showed a higher percentage of their time in SB than girls, and a reverse trend in the evening.

Table 1

Summary of Children's Characteristics Regarding Age, Body Weight, Overweight Status, and Behaviors

\begin{tabular}{|c|c|c|c|c|c|c|}
\hline \multirow[b]{2}{*}{ Characteristic } & \multicolumn{2}{|c|}{ Boys $(n=56)$} & \multicolumn{2}{|c|}{ Girls $(n=47)$} & \multicolumn{2}{|c|}{ Total $(N=103)$} \\
\hline & $M$ & $S D$ & $M$ & $S D$ & $M$ & $S D$ \\
\hline Age (years) & 9.91 & 0.86 & 9.94 & 0.72 & 9.93 & 0.80 \\
\hline Height (m) & 1.39 & 0.06 & 1.39 & 0.08 & 1.37 & 0.07 \\
\hline Body mass (kg) & 35.7 & 7.0 & 35.6 & 8.0 & 35.6 & 7.4 \\
\hline BMI $\left(\mathrm{kg} / \mathrm{m}^{2}\right)$ & 18.29 & 2.69 & 18.20 & 2.87 & 18.25 & 2.76 \\
\hline Overweight & \multicolumn{2}{|c|}{$18(32 \%)$} & \multicolumn{2}{|c|}{$16(34 \%)$} & \multicolumn{2}{|c|}{$34(33 \%)$} \\
\hline$t_{\mathrm{MVPA}}(\mathrm{min})^{\dagger}$ & 36 & 24 & 18 & 10 & 28 & 21 \\
\hline$t_{\mathrm{SB}}(\mathrm{min})^{*}$ & 704 & 44 & 727 & 35 & 712 & 41 \\
\hline
\end{tabular}

Note-In the Overweight row only, the figures denote number (and percentage) of overweight children. BMI, body mass index; $t_{\mathrm{MVPA}}$, time spent at moderate to vigorous physical activity; $t_{\mathrm{SB}}$, time spent at sedentary behavior. ${ }^{*}$ Significant difference between boys and girls $(p<.01)$. †Significant difference between boys and girls $(p<.0001)$. 
Table 2

Intraclass Reliability Coefficients ( $R \mathrm{~s})$ and $95 \%$ Confidence Intervals (CIs) for 1-Day and 3-Day Monitoring

\begin{tabular}{cccccc}
\hline & \multicolumn{2}{c}{$t_{\text {MVPA }}$} & & \multicolumn{2}{c}{$t_{\mathrm{SB}}$} \\
\cline { 2 - 3 } \cline { 5 - 6 } & 1 Day & 3 Days & & 1 Day & 3 Days \\
\hline$R$ & 0.50 & 0.75 & & 0.35 & 0.62 \\
CI & $0.39-0.61$ & $0.66-0.82$ & & $0.23-0.48$ & $0.48-0.73$ \\
\hline
\end{tabular}

Note $-t_{\mathrm{MVPA}}$, time spent at moderate to vigorous physical activity; $t_{\mathrm{SB}}$, time spent at sedentary behavior.

\section{DISCUSSION}

The purpose of this study was to examine the hourly variation in and the interplay between physical activity and SB among children and to discuss the relevance of the use of the principal component analysis (PCA) method in the underlying of key time periods for physical activity interventions for children. The main results show that overall, physical activity is inversely related to sedentary behavior. The current data do not agree with earlier statements which viewed physical activity and sedentary lifestyle as independent behaviors. Furthermore, when analyzed as a time function, ten components were extracted, with a competition between MVPA and SB among eight of them. The highest percentage of children's MVPA-time occurred in the afternoon whereas the highest percentage they spent being sedentary occurred in the morning hours.

To our knowledge, few studies (Durant et al., 1993; Mota et al., 2003; Trost et al., 2000) have analyzed the suitability of specific time periods in the explanation of habitual physical activity among children. And this is the first study which examines objectively measured physical activity and sedentary behavior levels together, as a whole and as a time function as well. The PCA provides ten distinct components that account for $80.4 \%$ of the variance. Only two of these factors (accounting for $8.5 \%$ of the variance) did not display competition between $t_{\mathrm{MVPA}}$ and $t_{\mathrm{SB}}$. An important period was obtained in the morning hours (Component 1). This first component was positively correlated with MVPA and negatively correlated with SB. This factor may correspond to the time when children were free for activity as they finished their breakfast or any other morning domestic activities. During school days, this factor could reflect the morning recess period hours. Another key component (Component 2) corresponding to the time after school or hours when children and their parents are reunited after a work or a school day. The next component (Component 3) though distinct from Component 2, seemed to anticipate this phenomenon and could be interpreted as the time of commuting to home. A particularity of the school time schedule in France is the incorporation of a free day in the middle of a whole school week, (and it is this free day which was used in the analyses). Thus, as parents may be at work, their children are generally sent to leisure centers, where they can spend the bulk of their time as if they were at school. In a study from our laboratory (unpublished data), up to one third of schoolchildren were sent to the community recreation centers on Wednesday where they are consigned to the care of trained instructors. Consequently, this free day presents many similarities with an authentic school day. This is the reason why Factors 2 and 3 could be considered as hours when children meet up with their parents. On the other hand, Components 4 and 5 including after lunch period, afternoon recess period and commuting to home are distinct. The remaining components may correspond to: the beginning of classes or leisure time activities (Component 6), lunch period (Component 7) and at bed hours (Component 8). The aforementioned components highlighted a balancing between MVPA and SB. Nevertheless the waking hour (Component 10) and the time of arrival at schools or centers (Component 9) did not show any opposition between the two variables. It may be hypothesized that on those times, there were lesser possibility than other periods to get mixed behaviors, and that is why these components (Components 9 and 10) explained the least variance. Our data suggested that when MVPA and SB were analyzed together a balanced relationship could be obtained most of the time. In terms of intervention strategies, these results lend support to some empirical and experimental studies, which reported that by decreasing sedentary behavior, physical activity may increase and favor the treatment of childhood obesity (Epstein et al., 2000; Epstein et al., 1997; Epstein et al., 1995; Epstein et al., 1991). Furthermore, the results mean that "one cannot be both physically active and sedentary at the same time, since increases in sedentary time reduced the amount of time available to be active" (Jago et al., 2005, p. 373). Even though Marshall, Biddle, Gorely, Cameron, and Murdey (2004) reported that physical activity and SB can compete but also coexist, it appears that when behaviors are assessed with an objective technique such as motion sensors, only the competitive (or noncompetitive) aspect during a given block of time can be brought out. Moreover, from an intervention point of view, data obtained using motion sensors supported by direct observations or questionnaires offer potential to determine what behaviors children engage in, when they are being active or inactive.

Shortly, morning hours (07:00AM-11:59AM) by explaining approximately $33.8 \%$ of the variance appeared to be of interest. Second key periods are the afternoon hours $(02: 00 \mathrm{PM}-05: 59 \mathrm{PM})$ followed by the evening time (06:00PM-09:00PM) which explained $19.8 \%$ and $18.2 \%$ of the total information, respectively. Noon hours

Table 3

Components and Their Contributions

\begin{tabular}{ccc}
\hline Component & Eigenvalue & Percentage of Variation \\
\hline 1 (10:00 AM-11:59 AM) & 5.3 & 18.8 \\
2 (06:00 PM-07:59 PM) & 3.7 & 13.3 \\
3 (05:00 PM-05:59 PM) & 2.4 & 8.5 \\
4 (03:00 PM-04:59 PM) & 2.2 & 7.8 \\
5 (01:00 PM-02:59 PM) & 1.9 & 7.0 \\
6 (09:00 AM-09:59 AM) & 1.8 & 6.5 \\
7 (12:00 PM-12:59 PM) & 1.4 & 5.1 \\
8 (08:00 PM-08:59 PM) & 1.4 & 4.9 \\
9(08:00 AM-08:59 AM) & 1.2 & 4.3 \\
10(07:00 AM-08:59 AM) & 1.2 & 4.2 \\
\hline
\end{tabular}


$\mathrm{t}_{\mathrm{SB}}\left(\%\right.$ total $\left.\mathrm{t}_{\mathrm{SB}}\right)$

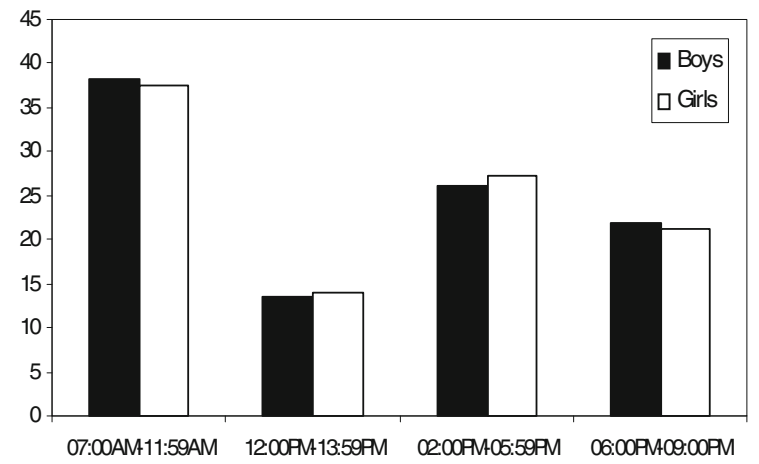

Aggregate time period

Figure 1. Percentage of time spent in sedentary behavior according to the total daily period.

(12:00PM-1:59PM) explained the least variance (8.6\%). Even though, comparison with other studies should be made with caution, our findings agree with those of Janz, Golden, Hansen, and Mahoney (1992). These authors reported that the most active time periods range from 09:00AM-11:59AM and from 04:00PM-05:59PM. However, from the present data, it cannot be stated absolutely that children were most active during these time frames. At best, the current results suggested that these periods are important to be considered. Nonetheless, when looking at the relative values of physical activity $\left(\% t_{\mathrm{MVPA}}\right)$ and physical inactivity $\left(\% t_{\mathrm{SB}}\right)$, as shown in figures 1 and 2 , an important moment of MVPA (more than $40 \%$ of their daily time at MVPA) was found in the afternoon hours, whereas the morning dealt with a relatively low level of physical activity (more than 35\% of their daily time in SB). Discrepancies with previous studies (Durant et al., 1993; Janz et al., 1992) may be linked to the time organization in France as compared to the USA; and also in methods used to describe activity parameters. Furthermore, noon hours are stable with regard to MVPA and SB as well, whereas sedentary pursuits may dominate in the evening.

From a public health standpoint, the data from the present study raise some perspectives in terms of intervention toward this schoolchildren population. Specially, playtime interventions or active transport interventions linked to the time frames found in this study may be important for physical activity engagement. This seems to be quite important since a number of them are almost obese or overweight (32\% of boys and $34 \%$ of girls). It can be hypothesized that targeting the reduction of SB in morning hours may be an appropriate way to improve children's physical activity. For this intervention purpose, further studies should be necessary to identify what children are doing specifically in these hours in order to propose other more attractive active alternatives. However, as to give some examples, it can be of interest to develop active commuting strategies (Cooper, Page, Foster, \& Qahwaji, 2003; Timperio et al., 2006; Tudor-Locke, Ainsworth, \& Popkin, 2001) - which assumes an implication of families and the community - create fun and active playgrounds for school recess activities or modify the duration of the morning recess (Guinhouya, Hubert, Dupont, \& Durocher, 2005; Ridgers \& Stratton, 2005; Zask, van Beurden, Barnett, Brooks, \& Dietrich, 2001) and so on. There appears also a need to sensitize parents about leisure centers and to endorse these centers with respect to their significance in the promotion of a healthy physical activity. Nevertheless, as empirical data do not exist to show the importance of these recreation centers in the French context, studies are needed to analyze this topic and to provide basis to policy makers. But, collectively, the present data seem to suggest that school-based and family-based programs may be adequate for these children.

The methodological approach used in the present study (principal component analysis) is rarely used in the literature to examine human behaviors. Nevertheless, it provides an indication of important times in a child's day for physical activity, which may be relevant in the choice of prevention decision and the development of health promotion interventions. Clearly, such a method enables a better organization of data for further studies and offers the first tools for prevention decision as far as the theoretical value and the clinical resonance of the components were attested. From previous accelerometry studies (Mota et al., 2003; Trost et al., 2000), only 2 to 4 components were extracted compared to the 10 of the present study. Two main reasons could explain this disagreement. First, in those studies, MVPA was analyzed solely whereas it was coupled with SB in this one. Second, the cut-point used in the present study to describe MVPA was different from that of previous authors. In fact, Guinhouya et al. (2006) recently highlighted comparability problems between studies when different thresholds were used. However, though caution should be exercised when interpreting the components and the necessity of further explicative analysis, the PCA may be useful in the description of children's behaviors, particularly for the identification and the targeting of adequate time periods of activity and/or inactivity.

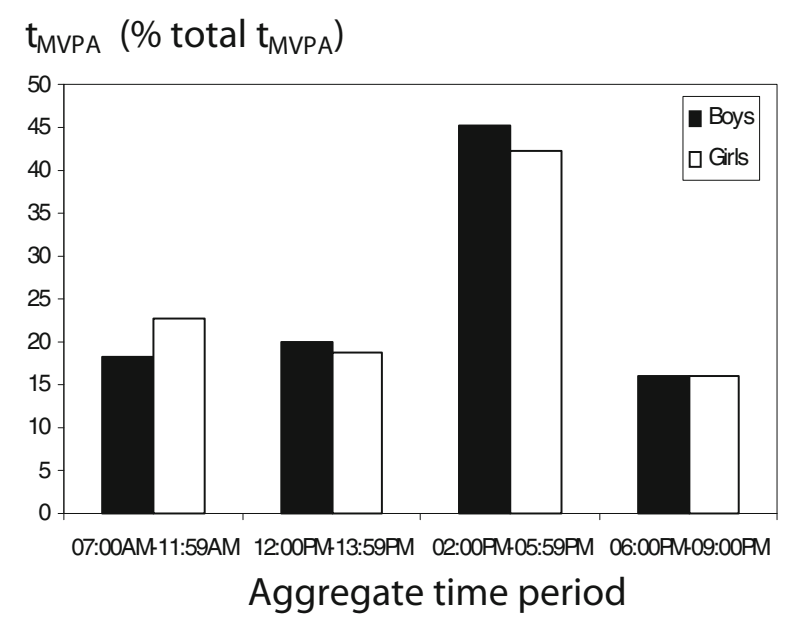

Figure 2. Percentage of time spent in moderate-to-vigorous physical activity according to the total daily period. 
There are a few limitations to this study. One of them is the grouping of data obtained on school days with those of the free day. This can make interpretation difficult or confusing and thus hamper the subtlety in interpreting obtained factorial axes. Nevertheless, putting data together remains a reasonable way to observe properly the reality of children's behaviors by increasing data reliability (Table 2). A second limitation is related to the epoch definition for data collection. Indeed, as reported by Nilsson, Ekelund, Yngve, and Sjöström (2002), there may be discrepancies in results when using a 5-sec epoch as compared to a 60 -sec one. Indeed, as children's activity occurs intermittently with short burst of intense activity interspersed with periods of rest (Bailey et al., 1995), it may be that a 60 -sec interval for data collection underestimate vigorous episodes of children's activity and reduce the potential for data to express accurately their overall MVPA. Nevertheless, increasing the time resolution as it can be suggested when attempting to assess with a high accuracy the vigorous episodes of children's activities, decrease the possible length of monitoring, due to the fixed memory size of older models of the ActiGraph. For example, a measurement with 10-sec epochs and simultaneous frequency recording allows a sampling of $44.4 \mathrm{~h}$, which may not be enough. In addition, the use of a PCA carries a risk of subjectivity that can be relieved or ruled out only by a certain level of practice of this method and the theoretical knowledge of children's behavior and environment. There is also a need to be familiar with exploration techniques used to gather and analyze data. Finally, it would be interesting to broaden the present results with a direct observation of children, in order to depict what they do during their active and/or inactive times. Such an approach may help to increase the achievement of interventions.

Finally, this study highlights an inverse relationship between objectively measured MVPA and SB as a time function, which offer some pertinent time frames for the implementation of prevention strategies. The use of a PCA enables a better organization of data, and consequently to gather the salient traits of a child behavior. Particularly, efficacy of interventions targeting the morning hours (07:00 AM-11:59 AM) and the afternoon period (02:00 PM-05:59 PM) warrants attention.

\section{AUTHOR NOTE}

The authors are indebted to the children of Chopin and Taine elementary schools (Villeneuve d'Ascq) and Jean Monnet primary school (Illies) and to their parents for their enthusiastic participation. We give thanks to the teachers. We also thank Pambou Ange and an anonymous reviewer for valuable comments that helped to improve the manuscript. Correspondence concerning this article should be addressed to $\mathrm{H}$. Hubert, LEM-MOSCADES, Institute of Engineering in Health of Lille, University of Lille 2, 42, rue Ambroise Paré, F-59120 Loos, France (e-mail: herve.hubert@univ-lille2.fr).

\section{REFERENCES}

Bailey, R. C., Olson, J., Pepper, S. L., Porszasz, J., Bartow, T. J., \& Cooper, D. M. (1995). The level and tempo of children's physical activities: An observational study. Medicine \& Science in Sports \& Exercise, 27, 1033-1041.

Biddle, S. J. H., \& Gorely, T. (2005). Couch kids: Myth or reality. The Psychologist, 18, 276-279.
Biddle, S. J. H., Gorely, T., \& Stensel, D. (2004). Health-enhancing physical activity and sedentary behaviour in children and adolescents. Journal of Sports Sciences, 22, 679-701.

Cole, T. J., Bellizi, M. C., Flegal, K. M., \& Dietz, W. H. (2000). Establishing a standard definition for child overweight and obesity worldwide: International survey. British Medical Journal, 320, 12401243.

Cooper, A. R., Page, A. S., Foster, L. F., \& Qahwaji, D. (2003). Commuting to school: Are children who walk more physically active? American Journal of Preventive Medicine, 25, 273-276.

Dietz, W. H. (1996). The role of lifestyle in health: The epidemiology and consequences of inactivity. Proceedings of the Nutrition Society, $\mathbf{5 5}, 829-840$.

Durant, R. H., Baranowski, T., Davis, H., Rhodes, T., Thompson, W. O., Greaves, K., \& Puhl, J. (1993). Reliability and variability of indicators of heart-rate monitoring in children. Medicine \& Science in Sports \& Exercise, 25, 389-395.

Epstein, L. H., Paluch, R. A., Gordy, C. C., \& Dorn, J. (2000). Decreasing sedentary behaviors in treating pediatric obesity. Archives of Pediatrics \& Adolescent Medicine, 154, 220-226.

Epstein, L. H., Saelens, B. E., Myers, M. D., \& Vito, D. (1997). Effects of decreasing sedentary behaviors on activity choice on obese children. Health Psychology, 16, 107-113.

Epstein, L. H., Saelens, B. E., \& O’Brien, J. G. (1995). Effects of reinforcing increases in active versus decreases in sedentary behavior in obese children. International Journal Behavioral Medicine, 2, 41-50.

Epstein, L. H., Smith, J. A., Vara, L. S., \& Rodefer, J. S. (1991). Behavioral economic analysis of activity choice in obese children. Health Psychology, 10, 311-316.

Falgairette, G., Gavarry, O., Bernard, T., \& Hebbelinck, M. (1996). Evaluation of habitual physical activity from a week's heart rate monitoring in French school children. European Journal of Applied Physiology, 14, 153-161.

Fox, K. R. (2004). Childhood obesity and the role of physical activity. Journal of the Royal Society for the Promotion of Health, 14, 75-84.

Guinhouya, C. B., Hubert, H., Dupont, G., \& Durocher, A. (2005). The recess period: A key moment of prepubescents' daily physical activity? International Electronic Journal of Health Education, 8, 126-134.

Guinhouya, C. B., Hubert, H., Soubrier, S., Vilhelm, C., Lemdani, M., \& Durocher, M. (2006). Moderate-to-vigorous physical activity: Discrepancies in accelerometry-based cut-points. Obesity Research, 14, 774-777.

Jago, R., Baranowski, T., Thompson, J., Baranowski, D. J., \& Greaves, K. A. (2005). Sedentary behavior, not TV viewing, predicts physical activity among 3- to 7-year-old children. Pediatric Exercise Science, 17, 364-376.

Janz, K. F., Golden, J. C., Hansen, J. R., \& Mahoney, L. T. (1992). Heart rate monitoring of physical activity in children and adolescents: The Muscatine study. Pediatrics, 89, 256-261.

Marshall, S. J., Biddle, S. J. H., Gorely, T., Cameron, N., \& MurdeY, I. (2004). Relationships between media use, body fatness and physical activity in children and youth: A meta-analysis. International Journal of Obesity, 28, 1238-1246.

Marshall, S. J., Biddle, S. J. H., Sallis, J. F., McKenzie, T. L., \& ConwaY, T. L. (2002). Clustering of sedentary behaviors and physical activity among youth: A cross-national study. Pediatric Exercise Science, 14, 401-417.

McCann, S. E., Weiner, J., Graham, S., Freudenheim, J. L. (2001). Is principal component analysis necessary to characterize dietary behavior in studies of diet and disease? Public Health Nutrition, 4, 903-908.

Mota, J., Santos, P., Guerra, S., Ribeiro, J. C., \& Duarte, J. A. (2003). Patterns of daily physical activity during school days in children and adolescents. American Journal of Human Biology, 15, 547-553.

Nilsson, A., Ekelund, U., YngVe, A., \& Sıöström, M. (2002). Assessing physical activity among children with accelerometers using different time sampling intervals and placements. Pediatric Exercise Science, 14, 75-84.

Puyau, M. R., Adolph, A. L., Vohra, F. A., \& Butte, N. F. (2002). Validation and calibration of physical activity monitors in children. Obesity Research, 10, 150-157. 
Randall, E., Marshall, J. R., Graham, S., \& Brasure, J. (1991). High risk health behaviors associated with various dietary patterns. Nutrition \& Cancer, 16, 135-151.

Reilly, J. J., Coyle, J., Kelly, L., Burke, G., Grant, S., \& Paton, J. Y. (2003). An objective method of measurement of sedentary behavior in 3- to 4-year-olds. Obesity Research, 11, 1155-1158.

Rennie, K. L., Johnson, L., \& JebB, S. A. (2005). Behavioural determinants of obesity. Best Practice \& Research Clinical Endocrinology \& Metabolism, 19, 343-358.

Ridgers, N. D., \& Stratton, G (2005). Physical activity during school recess: The Liverpool Sporting Playgrounds Project. Pediatric Exercise Science, 17, 281-290.

Timperio, A., Ball, K., Salmon, J., Roberts, R., Giles-Corti, B. Simmons, D., ET AL. (2006). Personal, family, social, and environmental correlated of active commuting to school. American Journal of Preventive Medicine, 30, 45-51.

Trost, S. G., Pate, R. R., Freedson, P. S., Sallis, J., \& Taylor, W. C.
(2000). Using objective physical activity measures with youth: How many days of monitoring are needed? Medicine \& Science in Sports \& Exercise, 32, 426-431.

Tudor-Locke, C., Ainsworth, B. E., \& Popkin, B. M. (2001). Active commuting to school: An overlooked source of children's physical activity? Sports Medicine, 31, 309-313.

Ursin, G., Ziegler, R. G., Subar, A. F., Graubard, B. I., Haile, R. W., \& Hoover, R. (1993). Dietary patterns associated with low-fat diet in the national examination follow-up study: Identification of potential confounders for epidemiologic analyses. American Journal of Epidemiology, 137, 916-927.

Zask, A., van Beurden, E., Barnett, L., Brooks, L. O., \& Dietrich, U. C. (2001). Active school playgrounds-Myth or reality? Results of the "Move It Groove It" Project. Preventive Medicine, 33, 402-408.

(Manuscript received June 15, 2006;

revision accepted for publication September 4, 2006.) 